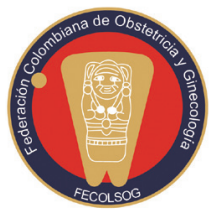

\title{
EVALUACIÓN DEL EFECTO DE UNA INTERVENCIÓN EDUCATIVA EN SÍFILIS CONGÉNITA A TRABAJADORES DE LA SALUD EN LA REGIÓN DEL URABÁ, COLOMBIA, 2008. ESTUDIO DE ANTES Y DESPUÉS
}

\section{Evaluating the effect of an educational intervention regarding congenital syphilis in healthcare-workers in the Urabá region of Colombia, 2008. A before and after study}

Liliana Isabel Gallego-Vélez, MD'; J Joaquín Guillermo Gómez-Dávila, MD, MSc²; John Jairo Zuleta-Tobón, MD, MSc ${ }^{3}$; Jesús Arnulfo Velásquez-Penagos, $\mathrm{MD}^{4}$; Silvia Elena Uribe-Bravo, Enf. ${ }^{5}$

Recibido: marzo 2/12 - Aceptado: noviembre 8/12

\section{RESUMEN}

Objetivo: evaluar el efecto de una intervención educativa al personal de salud en la adherencia a las guías de manejo de la sífilis gestacional (SG) y congénita (SC), el reporte oportuno y adecuado de los casos, y su percepción acerca de utilización de la prueba rápida para el diagnóstico de la sífilis. Materiales y métodos: se realizó un estudio de intervención con medición antes-después. Población: trabajadores en centros de primer nivel de atención del área rural de la población de Turbo ubicada en la región del Caribe colombiano, en zona limítrofe con Panamá, en el año 2008. La intervención con-

1 Ginecoobstetra. Docente Universidad de Antioquia. Medellín, Antioquia. ligave@gmail.com

2 Ginecoobstetra. Epidemiólogo clínico. Director Centro Nacer. Docente Universidad de Antioquia. Medellín, Antioquia.

3 Ginecoobstetra. Epidemiólogo clínico. Docente Universidad de Antioquia. Medellín, Antioquia.

4 Ginecoobstetra. Nacer, Universidad de Antioquia. Medellín, Antioquia.

5 Enfermera especialista en Auditoría en salud. Nacer, Universidad de Antioquia. Medellín, Antioquia. sistió en capacitación al personal de salud, entrega de material impreso y disponibilidad de prueba rápida para sífilis. Se evaluó la adquisición de conocimiento por parte del personal de salud intervenido y el efecto de la capacitación en el proceso de atención, la adherencia a las guías y la notificación y percepción de los trabajadores de la aplicación de la prueba rápida. La información se obtuvo directamente del personal de salud y de las historias clínicas de las mujeres que asistieron al programa. Análisis: para evaluar los cambios en la calificación de los exámenes de conocimiento se utilizó la prueba de rangos de Wilcoxon. Para la evaluación de los cambios en las proporciones de cumplimiento de recomendaciones se utilizó la prueba de chi cuadrado y se fijó un nivel de significancia estadística del 5\%.

Resultados: se evaluaron 533 historias clínicas y se capacitaron 129 personas. El reporte de casos de sífilis gestacional en Turbo mejoró de forma significativa ( $8 \%$ frente a $48 \%, \mathrm{p}=0,01$ ), al igual que el nivel de conocimientos en el personal de salud 
$(\mathrm{p}=0,002)$, la búsqueda (18\% frente a $53 \% \mathrm{p}=$ $0,001)$ y el tratamiento de contactos $(10 \%$ frente a $29 \%, \mathrm{p}=0,034)$ y el tratamiento adecuado de los recién nacidos (47\% frente a $85 \%, \mathrm{p}=0,03)$. $\mathrm{La}$ opinión del personal de salud acerca de la prueba rápida para sífilis fue favorable.

Conclusiones: la capacitación de los trabajadores del sector salud en el manejo de la sífilis congénita mejora la calidad de la atención a las gestantes y los recién nacidos con sífilis.

Palabras clave: sífilis gestacional, sífilis congénita, educación en salud, pruebas rápidas para sífilis.

\section{ABSTRACT}

Objective: Evaluating the effect of an educational intervention aimed at healthcare personnel regarding adherence to gestational syphilis (GS) and congenital syphilis (CS) management guidelines, early and suitable reporting of cases of GS and CS and their perception about using a rapid point-ofcare test for diagnosing syphilis.

Materials and methods: An intervention study was carried out involving before and after measurement. Population: staff working in first-level healthcare centres in a rural area attending the population of Turbo located in Colombia's Caribbean region, close to Panamá, during 2008. Such intervention consisted of training healthcare staff, delivering printed material and providing a rapid point-ofcare syphilis test. How effectively the healthcare staff had acquired such knowledge and the effect of the training given on attending patients was evaluated regarding adherence to the guidelines, notification and workers' perception of using the rapid test. Information was directly obtained from the healthcare staff and the clinical histories of females attending the programme.

Statistical analysis: A Wilcoxon signed-rank test was used for evaluating changes when scoring the test of their knowledge (before/after). A Chi ${ }^{2}$ test was used for evaluating changes in percentages regarding fulfilling the recommendations (statistical significance was fixed at 5\%).
Results: 533 clinical histories were evaluated and 129 people were trained. Reporting GS cases in Turbo improved significantly (8\% vs 48\%; p = $0.01)$, as well as the healthcare personnel's level of knowledge ( $\mathrm{p}=0.002)$, the search for $(18 \%$ vs $53 \%$; $\mathrm{p}=0.001)$ and treatment of contacts (10\% vs 29\%; $\mathrm{p}=0.034)$ and suitable treatment of the new-born (47\% vs $85 \% ; p=0.03)$. Healthcare personnel had a favourable opinion about the rapid syphilis test.

Conclusions: Training orientated towards resolving local problems improves the quality of healthcare attention provided for pregnant females and newborns babies suffering syphilis.

Key words: Gestational syphilis, congenital syphilis, healthcare education, rapid syphilis test.

\section{INTRODUCCIÓN}

La sífilis congénita (SC) es una enfermedad adquirida por lo general in utero, que resulta de la transmisión transplacentaria del Treponema pallidum de una madre infectada al fruto de la gestación, y es causa de abortos, muertes fetales y neonatales, bajo peso al nacer, prematurez, hidropesía, alteraciones neurológicas y óseas en los recién nacidos, entre otras $(1,2)$. En Colombia, las tasas de SC van en aumento, para el año 2010 la tasa fue de 3,08 casos por mil nacidos vivos -2.021 casos (3) entre 654.627 nacidos (4)-. En el departamento de Antioquia las tasas han disminuido levemente desde 2006, pero regiones como el Urabá antioqueño persisten con tasas más altas que las del promedio departamental. Según informes de la vigilancia epidemiológica de la SC realizada por la Secretaría Seccional de Salud de Antioquia (SSSA), la falta de realización o el ingreso tardío de la mujer afectada al control prenatal, las deficiencias del personal de salud para diagnosticar y tratar adecuadamente la sífilis gestacional y congénita, y el inadecuado seguimiento de los casos son los principales factores asociados a la presencia de SC en la región de Urabá. En el año 2007, la SSSA evaluó la calidad de los programas de salud reproductiva en Turbo, uno de 
los municipios de Urabá, y encontró solo un 40\% de cumplimiento de los estándares de calidad de atención en el control prenatal $(\mathrm{CP})$, por ejemplo, en un 25\% de las historias clínicas de CP no se evidenció la ejecución de las dos serologías para el tamizaje de sífilis (5), que por protocolo se deben ofrecer a todas las gestantes $(6,7)$. Estos hallazgos motivaron la realización de una intervención educativa al personal de salud en el municipio de Turbo, que se llevó a cabo en julio de 2008 con el ánimo de incrementar la calidad del servicio en el manejo de la sífilis gestacional y congénita. El objetivo de la investigación fue evaluar el efecto de esta intervención educativa en cuanto a: los conocimientos del personal de salud, la adherencia a las guías de manejo de la sífilis gestacional (SG) y congénita, el reporte oportuno y adecuado de los casos de SC, y la percepción del personal acerca de la utilización de la prueba rápida para el diagnóstico de la sífilis.

\section{MATERIALES Y MÉTODOS}

Se realizó un estudio cuasiexperimental con medición de antes-después. La población de intervención fueron los profesionales de salud responsables de realizar la atención de la población obstétrica de las siete instituciones de primer nivel de atención del área rural del municipio de Turbo, en la región del Urabá en el Caribe colombiano, cerca de Panamá. Estas instituciones atienden pacientes del aseguramiento contributivo para trabajadores y pacientes subsidiados por el Estado. La intervención se llevó a cabo en julio de 2008. Se realizó un muestreo consecutivo a los 129 trabajadores delegados por los directivos de las diferentes instituciones, no se tuvieron criterios de exclusión.

La unidad de análisis del efecto de la intervención fueron los pacientes atendidos en los centros seleccionados durante el año 2008. Para la selección de las historias se hizo un muestreo aleatorio simple: a partir del número total de pacientes atendidas en el periodo en cada institución, se generaron listados de números aleatorios. Para la evaluación de adquisición de conocimiento se tuvieron en cuenta los exámenes de todos los asistentes a la capacitación. Se calculó un tamaño de 442 historias para la evaluación de la calidad de la atención con base en una diferencia esperada del 13\% en la adherencia al protocolo de atención, con un poder del $80 \%$ y un nivel de confianza del 5\%, asumiendo una adhesión antes de la intervención del 27\%, cifra identificada en las evaluaciones previas hechas por la SSSA. Esta cifra se incrementó en 20\% para compensar la falta de datos que pudiera ocurrir en algunas variables.

Procedimiento. En los diferentes centros de atención se incluyeron en el estudio las historias que por orden de ubicación física dentro del archivo -que está organizado de forma ascendente según el número de historia- correspondieran al número generado en la secuencia aleatoria. Se excluyeron las historias con información incompleta y se reemplazaron por la siguiente cuando no se encontraba la elegida. Adicional a estas historias elegidas por azar, para algunos análisis se adicionaron todas las de casos con diagnóstico de SG y de SC.

Se elaboró una línea de base donde se recabó información sobre el reporte de casos, la calidad del diagnóstico y el seguimiento de las pacientes atendidas durante los seis meses previos (fase 1) a la intervención. Luego se realizó la intervención y, posteriormente, se hizo seguimiento en los aspectos antes mencionados en los seis meses siguientes (fase 2) a la intervención. La evaluación antes y después de la intervención la hizo una enfermera con entrenamiento en el tema mediante la confrontación de los hallazgos de las historias clínicas contra las recomendaciones que dan los protocolos del Ministerio de la Protección Social y el Instituto Nacional de Salud de Colombia del año 2007 (6) sobre el proceso de atención a las gestantes en general, a las gestantes con SG y a los recién nacidos con SC.

La intervención consistió en realización de talleres de capacitación ${ }^{1}$, entrega de material impreso para recordación y distribución de pruebas rápidas.

\footnotetext{
La intervención se realizó en el mes de julio de 2008, los talleres tuvieron una duración de tres días, y se dejó material impreso para el personal; asimismo, la línea telefónica estuvo disponible todo el tiempo.
} 
Se hicieron jornadas de cuatro horas de duración en grupos de 22 personas, coordinadas por dos médicos especialistas en obstetricia. La metodología de la capacitación fue con charlas magistrales, conversatorios y talleres. Los temas de capacitación fueron: diagnóstico y tratamiento de SG y SC, uso de la prueba rápida, estandarización del proceso de diligenciamiento de la ficha de notificación y del reporte al Sistema de Vigilancia Epidemiológica (sivigila). El material impreso contenía la guía de manejo de SG y SC con los flujogramas de manejo y la guía de realización e interpretación de la prueba rápida para sífilis. La prueba rápida utilizada fue SD Bioline Syphilis 3.0®, la cual se distribuyó a las auxiliares de enfermería de los distintos puestos y centros de salud, y a los coordinadores de los laboratorios clínicos. Durante el tiempo de la investigación se puso a disposición del personal de salud de Turbo una línea telefónica móvil para asesoría técnica en eventos de dudas en el diagnóstico, tratamiento o seguimiento de los casos.

Para asignar el diagnóstico de SG y SC se confirmó que la información consignada en la historia clínica cumpliera con las definiciones del protocolo del Ministerio de la Protección Social y el Instituto Nacional de Salud de Colombia del año 2007, donde el caso confirmado de sífilis gestacional corresponde a: toda mujer gestante, puérpera o con aborto reciente, con prueba no treponémica (VDRL o RPR) reactiva mayor o igual a 1:8 diluciones, o en menores diluciones con prueba treponémica positiva, y el caso confirmado de sífilis congénita corresponde al fruto de la gestación (aborto, mortinato o nacido vivo) de madre con sífilis gestacional sin tratamiento o con tratamiento inadecuado, independientemente que el recién nacido presente o no signos de enfermedad y del resultado de las pruebas no treponémicas en este.

Se evaluaron los conocimientos, la adherencia a las guías de manejo de la sífilis gestacional (SG) y congénita, el reporte oportuno y adecuado de los casos de SC, y la percepción sobre la utilización de la prueba rápida para el diagnóstico de la sífilis.
Para la evaluación de la adquisición de conocimientos por parte del personal de salud se hicieron exámenes escritos inmediatamente antes y cuatro meses después de la jornada de capacitación. Estas pruebas consistieron en quince preguntas de selección múltiple, con cuatro distractores, sobre aspectos prácticos del diagnóstico, tratamiento y notificación de SG y SC, diferentes según las competencias esperadas de personal médico y de enfermería, diseñadas por el equipo investigador. La calificación se hizo en una escala de 0 a 100 en representación del porcentaje de respuestas correctas.

Para la evaluación del efecto de la intervención en la adherencia a las guías sobre el proceso de atención, se evaluaron: el reporte de casos, la calidad del diagnóstico y el seguimiento de las pacientes atendidas. La adherencia a las recomendaciones se calculó como el porcentaje de casos en los cuales se identificaba en la historia el cumplimiento de las recomendaciones en el total de historias con la información. Se tuvieron en cuenta el número de días transcurridos entre la orden de realizar el examen de tamizaje y la evaluación del resultado por parte del médico, el número de días transcurridos entre la orden de la prueba y el inicio del tratamiento, la utilización de pruebas confirmatorias cuando estuviera indicado, el cumplimiento de los esquemas terapéuticos según la fase de la enfermedad, la búsqueda de compañeros potencialmente infectados.

Después de la intervención se hizo una encuesta a los usuarios de la prueba rápida para evaluar su percepción. Además, se evaluó el grado de dificultad tenido con la aplicación de la prueba rápida y los obstáculos para tomar decisiones con base en ella.

Para el análisis estadístico se creó una base de datos en Access y se utilizó el programa SPSS versión 17. Las variables cuantitativas discretas se presentan con mediana y percentiles (p) 25 y 75 . Las variables cualitativas se presentan como número absoluto y relativo. Para evaluar los cambios en la calificación de los exámenes de conocimiento se utilizó la prueba de rangos de Wilcoxon, dado que dichos cambios son medidos en los mismos individuos, por tanto, 
se consideran correlacionados. Para la evaluación de los cambios en las proporciones de cumplimiento de recomendaciones se utilizó la prueba de chi cuadrado porque la medición de los desenlaces se realizó en poblaciones diferentes, por tanto, se consideran independientes. Las pruebas fueron de dos colas y se fijó un nivel de significancia estadística del 5\%.

La investigación contó con la aprobación del comité de Bioética del Instituto de Investigaciones Médicas de la Facultad de Medicina de la Universidad de Antioquia, y el Comité de Ética Humana de la Facultad de Salud de la Universidad del Valle. Las pacientes aceptaron la realización de la prueba rápida por medio de un consentimiento informado.

\section{RESULTADOS}

Se capacitó al 42\% del personal médico y de enfermería del municipio de Turbo (129/271). Previo a la capacitación presentaron el examen 95 personas y 82 después de la misma. En la tabla 1 se observan los resultados de la evaluación antes-después discriminada según el tipo de profesión, con diferencias estadísticamente significativas en el nivel de conocimientos.
En el periodo de estudio se presentaron 48 casos de SG y 19 de SC en la fase 1, y 54 casos de SG y 20 de SC en la fase 2. Se encontró un incremento en el reporte de casos tanto de SG como de SC al sistema nacional de vigilancia, pero el de esta última no fue estadísticamente significativo (tabla 2). Los casos no reportados al Sistema Nacional de Vigilancia en Salud Pública (sivigila) pero encontrados en la investigación reflejan el subregistro de la notificación, la cual pasó de 72 a 52\% para la SG, y de 79 a 55\% para la SC.

Para evaluar la adherencia a las recomendaciones de la guía de atención se analizaron 277 historias en la fase 1 y 256 en la fase 2. En la fase 1 la mediana del número de días transcurridos entre la orden de realizar el examen de tamizaje y la evaluación del resultado fue de 8 días (p 25-75: 5-17,5), y en la fase 2 fue de 6 días (p 25-75: 0-7), diferencia estadísticamente significativa $(\mathrm{p}<0,001)$.

Se encontraron 45 mujeres con serología a títulos bajos (1:2 o 1:4) que, según la guía nacional vigente al momento del estudio, requerían de una prueba treponémica confirmatoria, sin embargo, a $16(35,5 \%)$ de estas mujeres no se les realizó prueba

\begin{tabular}{|c|c|c|c|c|c|c|}
\hline \multicolumn{7}{|c|}{$\begin{array}{l}\text { Tabla } 1 . \\
\text { Conocimientos que los médicos y el personal de enfermería tenía antes y después de la } \\
\text { capacitación en sífilis gestacional y congénita }\end{array}$} \\
\hline \multirow{2}{*}{$\begin{array}{l}\text { Evaluación de } \\
\text { conocimientos } \\
\text { en sífilis }\end{array}$} & \multicolumn{2}{|c|}{ Personal de enfermería } & \multirow{2}{*}{$\begin{array}{l}\text { Valor } \\
\text { de p }\end{array}$} & \multicolumn{2}{|c|}{ Personal médico } & \multirow{2}{*}{$\begin{array}{l}\text { Valor } \\
\text { de p }\end{array}$} \\
\hline & Antes & Después & & Antes & Después & \\
\hline $\begin{array}{l}\text { No. de exámenes } \\
\text { presentados }\end{array}$ & 57 & 50 & - & 38 & 32 & - \\
\hline $\begin{array}{l}\text { Percentil } 50 \text { conocimientos } \\
\text { en el diagnóstico } \\
\text { (percentil } 25-75 \text { ) }\end{array}$ & $\begin{array}{c}33,3 \% \\
(0-33,3)\end{array}$ & $\begin{array}{c}66,7 \% \\
(33,3-100)\end{array}$ & $<0,001$ & $\begin{array}{c}70 \% \\
(40-80)\end{array}$ & $\begin{array}{c}100 \% \\
(80-100)\end{array}$ & 0,002 \\
\hline $\begin{array}{l}\text { Percentil } 50 \text { conocimientos } \\
\text { en el manejo } \\
\text { (percentil } 25-75)\end{array}$ & $\begin{array}{c}33,3 \% \\
(0-33,3)\end{array}$ & $\begin{array}{c}66,7 \% \\
(33,3-100)\end{array}$ & 0,002 & $\begin{array}{c}60 \% \\
(40-80)\end{array}$ & $\begin{array}{c}80 \% \\
(65-100)\end{array}$ & $<0,001$ \\
\hline $\begin{array}{l}\text { Percentil } 50 \text { conocimientos } \\
\text { en la vigilancia epidemiológica } \\
\text { (percentil } 25-75 \text { ) }\end{array}$ & $\begin{array}{c}0,0 \% \\
(0-50)\end{array}$ & $\begin{array}{l}50,0 \% \\
(0-100)\end{array}$ & 0,007 & $\begin{array}{c}33,3 \% \\
(0-66,7)\end{array}$ & $\begin{array}{c}100 \% \\
(66,7-100)\end{array}$ & $<0,001$ \\
\hline
\end{tabular}

Se presenta el percentil 50 de la calificación obtenida para cada uno de los temas evaluados con sus respectivos percentiles 25 y 75 . La calificación va de 0 a 100 y representa el porcentaje de respuestas correctas. 
Tabla 2.

Notificación de casos de sífilis gestacional y congénita antes y después de la intervención

\begin{tabular}{|l|c|c|c|}
\hline Casos & Fase 1 & Fase 2 & Valor de p \\
\hline Sífilis gestacional & $4 / 48(8 \%)$ & $26 / 54(48 \%)$ & $<0,01$ \\
\hline Sífilis congénita & $4 / 19(21 \%)$ & $9 / 20(45 \%)$ & 0,17 \\
\hline
\end{tabular}

Nota: en el numerador se presenta el número de casos de sífilis reportados al sivigiLA, y en el denominador el número total de casos (los encontrados por búsqueda activa más los reportados).

treponémica, 13 (29\%) no tenían dato ni de solicitud ni de resultado dentro de la historia, y solo a $16(35,5 \%)$ se les realizó la prueba confirmatoria: 3 de las 23 con dato en la fase $1(13 \%)$ y 13 de las 22 con dato en la fase $2(59 \%)(p=0,0018)$.

En las mujeres con sífilis gestacional que iniciaron control prenatal antes de la semana 29, el tratamiento fue adecuado y oportuno en 5 de los 17 casos (29\%) de la fase 1, y en 12 de los 24 casos (50\%) en la fase $2(\mathrm{p}=0,8)$.

Para los casos en los que se contó con información, el tratamiento de las gestantes con sífilis fue adecuado según el estadio de la enfermedad materna en 21 de los 29 casos $(72,4 \%)$ en la fase 1 , y en 31 de los 38 casos $(81,6 \%)$ en la fase $2(p=0,147)$.

Los 48 casos de SG de la fase 1 se captaron mediante VDRL. De los 54 casos de SG diagnosticados en la fase 2, se les realizó la prueba rápida a 17 (31,5\%), 3 de ellos para tamizaje y 14 para confirmación del diagnóstico.

En la fase 1 se buscaron 9 de 48 contactos de mujeres con diagnóstico de sífilis gestacional $(18,8 \%)$, y en la fase 2 se buscaron 29 de 54 (53,7\%) (p < 0,001). Independientemente del motivo -falta de búsqueda, falta de ubicación o rechazo del tratamiento-, en la fase 1 se trataron 5 de los 48 contactos $(10,4 \%)$ y en la fase 2 se trataron 16 de $54(29,6 \%)(p=0,034)$.

Con independencia de la fase de realización de la prueba, la mediana del número de días transcurridos entre la orden de la prueba y el inicio del tratamiento, entre los 29 casos con diagnóstico por VDRL que tenían el dato, fue de 1 día (p 25-75: 1-7), y para los 11 casos con diagnóstico por prueba rápida que tenían el dato fue de 4 días (1-6) ( $p=0,68)$.

En la primera fase, 9 de los 19 casos de sífilis congénita (47\%) recibieron tratamiento adecuado, y en la segunda fase, 17 de $20(85 \%)(p=0,03)$. En la primera fase se encontró plan de seguimiento del recién nacido consignado en la historia clínica del 31,5\% (6 de 19), y en la segunda fase del 35\% (7 de 20) $(\mathrm{p}=0,8)$.

Al final del periodo de estudio se hizo una encuesta sobre la opinión del personal de salud acerca de la prueba rápida, la cual fue diligenciada por 32 personas que tuvieron oportunidad de efectuarla (15 enfermeras o auxiliares de enfermería, 16 médicos y una bacterióloga). Al 56\% la realización de la prueba le pareció muy fácil, al 34\% fácil, y el 10\% restante no respondió. La interpretación de los resultados fue muy fácil para el 66\% y fácil para el 34\%. Informaron que a pesar de contar con un resultado oportuno, el tratamiento con penicilina benzatínica solo lo podían administrar en el hospital local por la directriz de realizar prueba de sensibilidad previa en servicios de urgencias. Adicionalmente, en el 30\% de los centros de salud donde se diagnosticaron casos por prueba rápida no se contó con la presencia permanente del médico.

\section{DISCUSIÓN}

En la presente investigación se presenta el efecto de una intervención educativa realizada al personal de salud que labora en centros de primer nivel de atención del área rural de la población de Turbo so- 
bre el proceso de atención de las gestantes con sífilis gestacional y los recién nacidos con sífilis congénita. Se encontró un efecto favorable y significativo en la apropiación del conocimiento por el personal de salud, y en la adherencia a los protocolos de manejo en cuanto a la solicitud de prueba confirmatoria, búsqueda y tratamiento de los contactos, y tratamiento de la sífilis congénita. También se encontró un importante subregistro de los casos de sífilis.

Según informes de la Organización Panamericana de la Salud (OPS), el subregistro de casos de sífilis es común en Latinoamérica y esto hace que la magnitud del problema no se perciba en sus reales dimensiones y limite las estrategias y actividades de abogacía (8). En este estudio se logró incrementar la notificación al ente oficial, lo cual contribuye a tener una visión más fidedigna de la magnitud del problema.

Respecto a la notificación del contacto, en un estudio en Nairobi (9) se implementó la estrategia de tamizaje y tratamiento en el sitio de atención del control prenatal, logrando un aumento del 60 al $100 \%$ en el tamizaje, un aumento en el porcentaje de tratamiento materno adecuado del 9 al 87\%, y se logró tratar casi al 50\% de los contactos. En una experiencia en Kenia, basados en un sistema piloto de notificación, se logró que el 70\% de los compañeros de las pacientes positivas para sífilis recibieran tratamiento institucional (10). En nuestro estudio no fue posible reproducir estos resultados alentadores, pues, aunque hubo un incremento en la proporción de tratamientos maternos adecuados, no alcanzaron significación estadística y, si bien hubo un aumento significativo en la búsqueda y el tratamiento de los contactos, la proporción sigue siendo subóptima, lo cual se puede explicar por las condiciones propias de la población y el lugar donde se realizó, o por la falta de continuidad de la intervención.

Uno de los principales objetivos de la implementación de la prueba rápida para tamizaje es mejorar la oportunidad en el tratamiento de las gestantes y así evitar la infección fetal y neonatal. En un estudio realizado Suráfrica se encontró que el diagnóstico y el tratamiento correcto mejoraron cuando se utilizó la prueba rápida para sífilis en el sitio de atención (11). En nuestro estudio, sin embargo, no se logró disminuir el número de días transcurridos entre la evaluación del resultado de la prueba positiva y el inicio del tratamiento por la falta de médico permanente en los centros de salud donde se diagnosticaron casos por prueba rápida. Igualmente, la forma en que se trabaja en la región disminuye artificialmente el tiempo que transcurre entre el diagnóstico con el VDRL y el tratamiento, porque el VDRL se procesa en el hospital y los resultados llegan a los centros de salud con el médico que se desplaza a realizar la atención, lo que hace que la prescripción del tratamiento se dé el mismo día en que se consigna el diagnóstico.

Según la encuesta realizada al final de la intervención al personal participante, una de las explicaciones para que en nuestro estudio las mujeres no se beneficiaran de la realización de la prueba rápida es la directriz existente en Colombia según la cual los tratamientos con penicilina solo se pueden ordenar bajo prescripción médica, previa realización de una prueba de sensibilidad, la cual no se puede hacer en centros ni puestos de salud. La Organización Mundial de la Salud (OMS) promulga que se deben eliminar las barreras de acceso al tratamiento inmediato, una vez se realice el diagnóstico por los agentes de salud que realicen el control prenatal $(12,13)$. Aunque la recomendación colombiana pretende prevenir daños por el uso inadecuado del antibiótico, es conveniente poner en la balanza el hecho de que un 25\% de los embarazos complicados con sífilis culminan en aborto o mortinato (14), en cambio, la frecuencia estimada de anafilaxis severa que amenaza la vida asociada a la aplicación de penicilina es de 0,01 a $0,05 \%(15,16)$. Según esto, es conveniente que en Colombia se reevalúe la normatividad en cuanto a la aplicación de penicilina en áreas rurales, dado que el riesgo de muerte perinatal por sífilis es mayor que el riesgo de anafilaxia por la aplicación de penicilina.

En este estudio solo el 35\% de las gestantes con títulos bajos tuvieron prueba treponémica, y 
en muchas historias no se logró encontrar el dato, esto indica que muchos de los diagnósticos de sífilis no se están confirmando. Entre los casos de sífilis gestacional diagnosticados con prueba rápida su principal utilidad fue en la confirmación del caso. La Organización Mundial de la Salud propone el uso de pruebas rápidas para confirmar la sífilis en gestantes con pruebas no treponémicas reactivas donde se dispone de estas últimas para tamizaje (17). Esto tiene implicaciones prácticas dado que en nuestro medio el FTA-ABS y otras pruebas treponémicas solo se realizan en laboratorios de referencia. Las pruebas rápidas tienen en general alta sensibilidad y especificidad, la OMS comparó su rendimiento con TPHA/TPPA reportando sensibilidad de 84,5$97,7 \%$ y especificidad de $92,8-98 \%(18,19)$.

Nuestro estudio presenta varias limitaciones: la calidad de la información consignada en las historias clínicas es deficiente lo que le resta validez a algunos de los resultados; la fragmentación del proceso de atención de las pacientes; la falta de continuidad en el tiempo en la intervención, y la dificultad para modificar situaciones estructurales y de normatividad limitan el real alcance de la propuesta.

Para el control de la sífilis congénita se requiere un programa adecuadamente planeado y monitorizado, que tenga en cuenta al personal que lo maneja (14). La evaluación del cumplimiento de las estrategias propuestas en el plan de eliminación de la sífilis congénita y en las guías de manejo requiere de auditoría de los procesos y de su aplicación en las pacientes con el fin de identificar las deficiencias sobre las cuales se deben establecer planes de mejoramiento continuo.

En Colombia se requieren más estudios con prueba rápida para sífilis con el fin de buscar las estrategias más efectivas para su implementación y para lograr eliminar las barreras en la aplicación inmediata de penicilina.

No es posible calcular la incidencia de sífilis gestacional porque no se cuenta con el total de gestantes del periodo en las instituciones participantes. La incidencia de SC en Turbo para el año de 2008 fue de 12 casos por 1000 nacidos vivos (39 casos entre 3249 nacidos vivos).

\section{CONCLUSIONES}

La capacitación de los trabajadores de la salud en el manejo y reporte de la sífilis congénita mejora la adherencia a las guías, la calidad de la vigilancia epidemiológica y de la atención a las gestantes y los recién nacidos con sífilis.

Agradecimientos. A la Secretaría de Salud de Turbo; al ESE Hospital Francisco Valderrama, Turbo; a las IPS del municipio que participaron; a la doctora Mary Kamb, al doctor Nicholas Gaffga y a Jennifer Mark del US Centers for Disease Control and Prevention-Division of STD Prevention; Mary Hall, US Centers for Disease Control and Prevention-Division of Reproductive Health; al CEDETES - Universidad del Valle; Annar Diagnóstica Import Ltda.

\section{REFERENCIAS}

1. Ingall D, Sánchez P, Baker C. Syphilis. En: Remington JS, Klein JO. Infectious diseases of the fetus and newborn infant. 3 ed. Philadephia: W B Saunders; 2006. p. 545-80.

2. Berman SM. Maternal syphilis: pathophysiology and treatment. Bull World Health Organ 2004; 82:433-8.

3. Instituto Nacional de Salud. Notificación de eventos de interés en Salud Pública 2008-2010. Vigilancia y Control en Salud Pública - Publicaciones. [Visitado 2012 Oct 1]. Disponible en: http://190.26.202.205/ index.php?idcategoria $=94562$.

4. Departamento Administrativo Nacional de Estadística (DANE). Estadísticas vitales - Nacimientos por área y sexo, según departamento y municipio de residencia de la madre. [Visitado 2012 Oct 1]. Disponible en: https://www.dane. gov.co/index.php?option $=$ com_content\&view $=$ article\&id $=1043 \&$ Itemid $=119$.

5. Uribe S, Peña B. Informe final de auditoría de la calidad, maternidad segura, ESE Hospital Francisco Valderrama, Turbo. Secretaría Seccional de Salud de Antioquia; 2007. 
6. Instituto Nacional de Salud - Ministerio de Salud y la Protección Social. Protocolo de Sífilis congénita y gestacional. Bogotá: INS; 2007.

7. Instituto Nacional de Salud. Protocolo de vigilancia y control de sífilis gestacional y congénita. Grupo Enfermedades Transmisibles. Equipo Funcional Infecciones de Transmisión Sexual. Bogotá: INS; 2011.

8. Valderrama J. Metodología para estudios de subnotificación de sífilis en embarazadas. Organización Panamericana de la Salud. Washington, D.C: OPS; 2005.

9. Jenniskens F, Obwaka E, Kirisuah S, Moses S, Yusufali FM, Achola JO, et al. Syphilis control in pregnancy: decentralization of screening facilities to primary care level, a demonstration project in Nairobi, Kenya. Int J Gynaecol Obstet 1995;48:S121-8.

10. Deperthes BD, Meheus A, O'reilly K, Broutet N. Maternal and congenital syphilis programmes: case studies in Bolivia, Kenya and South Africa. Bull World Health Organ 2004;82:410-6.

11. Bronzan RN, Mwesigwa-Kayongo DC, Narkunas D, Schmid GP, Neilsen GA, Ballard RC, et al. On-site rapid antenatal syphilis screening with an immunochromatographic strip improves case detection and treatment in rural South African clinics. Sex Transm Dis 2007;34:S55-60.

12. Hawkes S, Miller S, Reichenbach L, Nayyar A, Buse K. Antenatal syphilis control: people, programmes, policies and politics. Bull World Health Organ 2004;82:417-23.
13. Regional initiative for elimination of Mother-tochild transmission of HIV and congenital syphilis in Latin America and the Caribbean. Publication No. OPS/FCH/HI/05-10.I C Pan American Health Organization; 2010.

14. The Global Elimination of Congenital Syphilis: Rationale and Strategy for Action. Geneva: World Health Organization; 2007.

15. Park MA, Li JT. Diagnosis and Management of Penicillin Allergy. Mayo Clin Proc 2005;80:405-10.

16. The International Collaborative Study of Severe Anaphylaxis. Risk of anaphylaxis in a hospital population in relation to the use of various drugs: an international study. Pharmacoepidemiol Drug Saf 2003;12:195-202.

17. The Use of Rapid Syphilis Tests. The Sexually Transmitted Diseases Diagnostics Initiative (SDI). Special Programme for Research \& Training in Tropical Diseases (TDR) sponsored by UNICEF / UNDP / World Bank/ WHO; 2006.

18. World Health Organization. The sexually transmitted diagnostics initiative (SDI): special programme for research and training in tropical diseases (TDR). Geneva: World Health Organization; 2003.

19. Herring AJ, Ballard RC, Pope V, Adegbola RA, Changalucha J, Fitzgerald DW, et al. A multi-centre evaluation of nine, rapid, point-of-care syphilis tests using archived sera. Sex Transm Infect 2006;82:v7-12. 